\title{
Symmetry-Based Tight Binding Modeling of Halide Perovskite Semiconductors
}

Soline BOYER-RICHARD ${ }^{1 *}$, Claudine KATAN ${ }^{2}$, Boubacar TRAORE ${ }^{2}$, Reinhard SCHOLZ ${ }^{3}$, Jean-Marc JANCU ${ }^{1}$, Jacky EVEN $^{I^{*}}$.

${ }^{1}$ UMR FOTON, CNRS, INSA-Rennes, F-35708 Rennes, France.

${ }^{2}$ Institut des Sciences Chimiques de Rennes, UMR 6226, CNRS, Université de Rennes 1, F-35042 Rennes, France

${ }^{3}$ Dresden Integrated Center for Applied Physics and Photonics Materials, Technische Universität Dresden, Germany

\section{AUTHOR INFORMATION}

\section{Corresponding Authors}

*soline.richard@insa-rennes.fr*jacky.even@insa-rennes.fr 


\section{Supporting information 1: Tight Binding Hamiltonians}

Basis functions:

- B orbitals: atom position $\boldsymbol{r}_{0}=(0,0,0):\left|S_{0}\right\rangle,\left|X_{0}\right\rangle,\left|Y_{0}\right\rangle,\left|Z_{0}\right\rangle$

- X orbitals: atom position $\boldsymbol{r}_{1}=\left(\frac{1}{2}, 0,0\right)$ a: $\left|S_{1}\right\rangle,\left|X_{1}\right\rangle,\left|Y_{1}\right\rangle,\left|Z_{1}\right\rangle$

- X orbitals: atom position $\boldsymbol{r}_{2}=\left(0, \frac{1}{2}, 0\right) a:\left|S_{2}\right\rangle,\left|X_{2}\right\rangle,\left|Y_{2}\right\rangle,\left|Z_{2}\right\rangle$

- X orbitals: atom position $\boldsymbol{r}_{3}=\left(0,0, \frac{1}{2}\right) a:\left|S_{3}\right\rangle,\left|X_{3}\right\rangle,\left|Y_{3}\right\rangle,\left|Z_{3}\right\rangle$

Atomic level energies:

- $E_{s 0}=\left\langle S_{0}|H| S_{0}\right\rangle$

- $E_{p 0}=\left\langle X_{0}|H| X_{0}\right\rangle=\left\langle Y_{0}|H| Y_{0}\right\rangle=\left\langle Z_{0}|H| Z_{0}\right\rangle$

- $E_{S 1}=\left\langle S_{1}|H| S_{1}\right\rangle=\left\langle S_{2}|H| S_{2}\right\rangle=\left\langle S_{3}|H| S_{3}\right\rangle$

- $E_{p 1}=\left\langle X_{\beta}|H| X_{\beta}\right\rangle=\left\langle Y_{\beta}|H| Y_{\beta}\right\rangle=\left\langle Z_{\beta}|H| Z_{\beta}\right\rangle$ where $\beta=1,2,3$ for each $\mathrm{X}$ atom.

Overlap integrals:

- $V_{s S}=\left\langle S_{0}\left|H_{\text {int }}\right| S_{\beta}\right\rangle$ where $\beta=1,2,3$ for each $\mathrm{X}$ atom.

- $V_{s 0 p 1}=\left\langle S_{0}\left|H_{\text {int }}\right| X_{1}\right\rangle=\left\langle S_{0}\left|H_{\text {int }}\right| Y_{2}\right\rangle=\left\langle S_{0}\left|H_{\text {int }}\right| Z_{3}\right\rangle$

- $V_{p 0 s 1}=\left\langle X_{0}\left|H_{\text {int }}\right| S_{1}\right\rangle=\left\langle Y_{0}\left|H_{\text {int }}\right| S_{2}\right\rangle=\left\langle Z_{0}\left|H_{\text {int }}\right| S_{3}\right\rangle$

- $V_{p p \sigma}=\left\langle X_{0}\left|H_{\text {int }}\right| X_{1}\right\rangle=\left\langle Y_{0}\left|H_{\text {int }}\right| Y_{2}\right\rangle=\left\langle Z_{0}\left|H_{\text {int }}\right| Z_{3}\right\rangle$

- $V_{p p \pi}=\left\langle Y_{0}\left|H_{\text {int }}\right| Y_{1}\right\rangle=\left\langle Y_{0}\left|H_{\text {int }}\right| Y_{3}\right\rangle=\left\langle Z_{0}\left|H_{\text {int }}\right| Z_{1}\right\rangle=\left\langle Z_{0}\left|H_{\text {int }}\right| Z_{2}\right\rangle=\left\langle X_{0}\left|H_{\text {int }}\right| X_{2}\right\rangle=\left\langle X_{0}\left|H_{\text {int }}\right| X_{3}\right\rangle$ 
Additional variables:

- $g_{x}=2 \cos \left(k_{x} \frac{a}{2}\right) ; g_{y}=2 \cos \left(k_{y} \frac{a}{2}\right) ; g_{z}=2 \cos \left(k_{z} \frac{a}{2}\right)$

- $h_{x}=2 i \sin \left(k_{x} \frac{a}{2}\right) ; h_{y}=2 i \sin \left(k_{y} \frac{a}{2}\right) ; h_{z}=2 i \sin \left(k_{z} \frac{a}{2}\right) ; h_{p}=\frac{1}{\sqrt{2}}\left(h_{x}+h_{y}\right)$

\begin{tabular}{|c|c|c|c|c|c|c|c|c|c|c|c|c|c|c|c|c|}
\hline & $\left|S_{0}\right\rangle$ & $\left|X_{0}\right\rangle$ & $\left|Y_{0}\right\rangle$ & $\left|Z_{0}\right\rangle$ & $\left|S_{1}\right\rangle$ & $\left|X_{1}\right\rangle$ & $\left|Y_{1}\right\rangle$ & $\left|Z_{1}\right\rangle$ & $\left|S_{2}\right\rangle$ & $\left|X_{2}\right\rangle$ & $\left|Y_{2}\right\rangle$ & $\left|Z_{2}\right\rangle$ & $\left|S_{3}\right\rangle$ & $\left|X_{3}\right\rangle$ & $\left|Y_{3}\right\rangle$ & $\left|Z_{3}\right\rangle$ \\
\hline$\left\langle S_{0}\right|$ & $E_{s 0}$ & 0 & 0 & 0 & $V_{s S} g_{x}$ & $V_{s 0 p 1} h_{x}$ & 0 & 0 & $V_{s s} g_{y}$ & 0 & $V_{s 0 p 1} h_{y}$ & 0 & $V_{s S} g_{z}$ & 0 & 0 & $V_{s 0 p 1} h_{z}$ \\
\hline$\left\langle X_{0}\right|$ & 0 & $E_{p 0}$ & 0 & 0 & $-V_{p 0 s 1} h_{x}$ & $V_{p p \sigma} g_{x}$ & 0 & 0 & 0 & $V_{p p \pi} g_{y}$ & 0 & 0 & 0 & $V_{p p \pi} g_{z}$ & 0 & 0 \\
\hline$\left\langle Y_{0}\right|$ & 0 & 0 & $E_{p 0}$ & 0 & 0 & 0 & $V_{p p \pi} g_{x}$ & 0 & $-V_{p 0 s 1} h_{y}$ & 0 & $V_{p p \sigma} g_{y}$ & 0 & 0 & 0 & $V_{p p \pi} g_{z}$ & 0 \\
\hline$\left\langle Z_{0}\right|$ & 0 & 0 & 0 & $E_{p 0}$ & 0 & 0 & 0 & $V_{p p \pi} g_{x}$ & 0 & 0 & 0 & $V_{p p \pi} g_{y}$ & $-V_{p 0 s 1} h_{z}$ & 0 & 0 & $V_{p p \sigma} g_{z}$ \\
\hline$\left\langle S_{1}\right|$ & $V_{s s} g_{x}$ & $V_{p 0 s 1} h_{x}$ & 0 & 0 & $E_{s 1}$ & 0 & 0 & 0 & 0 & 0 & 0 & 0 & 0 & 0 & 0 & 0 \\
\hline$\left\langle X_{1}\right|$ & $-V_{s 0 p 1} h_{x}$ & $V_{p p \sigma} g_{x}$ & 0 & 0 & 0 & $E_{p 1}$ & 0 & 0 & 0 & 0 & 0 & 0 & 0 & 0 & 0 & 0 \\
\hline$\left\langle Y_{1}\right|$ & 0 & 0 & $V_{p p \pi} g_{x}$ & 0 & 0 & 0 & $E_{p 1}$ & 0 & 0 & 0 & 0 & 0 & 0 & 0 & 0 & 0 \\
\hline$\left\langle Z_{1}\right|$ & 0 & 0 & 0 & $V_{p p \pi} g_{x}$ & 0 & 0 & 0 & $E_{p 1}$ & 0 & 0 & 0 & 0 & 0 & 0 & 0 & 0 \\
\hline$\left\langle S_{2}\right|$ & $V_{s s} g_{y}$ & 0 & $V_{p 0 s 1} h_{y}$ & 0 & 0 & 0 & 0 & 0 & $E_{s 1}$ & 0 & 0 & 0 & 0 & 0 & 0 & 0 \\
\hline$\left\langle X_{2}\right|$ & 0 & $V_{p p \pi} g_{y}$ & 0 & 0 & 0 & 0 & 0 & 0 & 0 & $E_{p 1}$ & 0 & 0 & 0 & 0 & 0 & 0 \\
\hline$\left\langle Y_{2}\right|$ & $-V_{s 0 p 1} h_{y}$ & 0 & $V_{p p \sigma} g_{y}$ & 0 & 0 & 0 & 0 & 0 & 0 & 0 & $E_{p 1}$ & 0 & 0 & 0 & 0 & 0 \\
\hline$\left\langle Z_{2}\right|$ & 0 & 0 & 0 & $V_{p p \pi} g_{y}$ & 0 & 0 & 0 & 0 & 0 & 0 & 0 & $E_{p 1}$ & 0 & 0 & 0 & 0 \\
\hline$\left\langle S_{3}\right|$ & $V_{s s} g_{y}$ & 0 & 0 & $V_{p 0 s 1} h_{z}$ & 0 & 0 & 0 & 0 & 0 & 0 & 0 & 0 & $E_{s 1}$ & 0 & 0 & 0 \\
\hline$\left\langle X_{3}\right|$ & 0 & $V_{p p \pi} g_{z}$ & 0 & 0 & 0 & 0 & 0 & 0 & 0 & 0 & 0 & 0 & 0 & $E_{p 1}$ & 0 & 0 \\
\hline$\left\langle Y_{3}\right|$ & 0 & 0 & $V_{p p \pi} g_{z}$ & 0 & 0 & 0 & 0 & 0 & 0 & 0 & 0 & 0 & 0 & 0 & $E_{p 1}$ & 0 \\
\hline$\left\langle Z_{3}\right|$ & $-V_{s 0 p 1} h_{z}$ & 0 & 0 & $V_{p p \sigma} g_{z}$ & 0 & 0 & 0 & 0 & 0 & 0 & 0 & 0 & 0 & 0 & 0 & $E_{p 1}$ \\
\hline
\end{tabular}

Characteristics at $M\left(\frac{1}{2}, \frac{1}{2}, 0\right)$ point

At $M$ point, the additional variables verify:

- $g_{x}=0, g_{y}=0, g_{x}=2 ; h_{x}=2 i, h_{y}=2 i, h_{z}=0 ; h_{p}=\frac{1}{\sqrt{2}}\left(h_{x}+h_{y}\right)=2 i \sqrt{2}$

To obtain a block diagonal matrix at $M$ point, the basis functions are the following:

- $M_{1}^{+}$functions: $\left|S_{0}\right\rangle,\left|S_{3}\right\rangle,\left|P_{M 1}\right\rangle=\frac{1}{\sqrt{2}}\left(\left|X_{1}\right\rangle+\left|Y_{2}\right\rangle\right)$

- $M_{5}^{-}$functions: $\left|X_{0}\right\rangle,\left|Y_{0}\right\rangle,\left|X_{3}\right\rangle,\left|Y_{3}\right\rangle,\left|S_{1}\right\rangle,\left|S_{2}\right\rangle$ 
- $M_{2}^{-}$functions: $\left|Z_{0}\right\rangle,\left|Z_{3}\right\rangle$

- $M_{2}^{+}$function: $\left|P_{M 2}\right\rangle=\frac{1}{\sqrt{2}}\left(\left|Z_{1}\right\rangle+\left|Z_{2}\right\rangle\right)$

- $M_{3}^{+}$function: $\left|P_{M 3}\right\rangle=\frac{1}{\sqrt{2}}\left(\left|Z_{1}\right\rangle-\left|Z_{2}\right\rangle\right)$

- $M_{4}^{+}$function: $\left|P_{M 4}\right\rangle=\frac{1}{\sqrt{2}}\left(\left|X_{1}\right\rangle-\left|Y_{2}\right\rangle\right)$

- $2 M_{5}^{+}$functions: $\left|Y_{1}\right\rangle,\left|X_{2}\right\rangle$

Block-diagonal tight binding Hamiltonian at $M\left(\frac{1}{2}, \frac{1}{2}, 0\right)$ point:

\begin{tabular}{|c|c|c|c|c|c|c|c|c|c|c|c|c|c|c|c|c|}
\hline & $\left|S_{0}\right\rangle$ & $\left|S_{3}\right\rangle$ & $\left|P_{M 1}\right\rangle$ & $\left|S_{1}\right\rangle$ & $\left|S_{2}\right\rangle$ & $\left|X_{0}\right\rangle$ & $\left|Y_{0}\right\rangle$ & $\left|X_{3}\right\rangle$ & $\left|Y_{3}\right\rangle$ & $\left|Z_{3}\right\rangle$ & $\left|Z_{0}\right\rangle$ & $\left|P_{M 2}\right\rangle$ & $\left|P_{M 3}\right\rangle$ & $\left|P_{M 4}\right\rangle$ & $\left|Y_{1}\right\rangle$ & $\left|X_{2}\right\rangle$ \\
\hline$\left\langle S_{0}\right|$ & $E_{s 0}$ & $V_{s S} g_{z}$ & $V_{s 0 p 1} h_{p}$ & 0 & 0 & 0 & 0 & 0 & 0 & 0 & 0 & 0 & 0 & 0 & 0 & 0 \\
\hline$\left\langle S_{3}\right|$ & $V_{s s} g_{z}$ & $E_{s 1}$ & 0 & 0 & 0 & 0 & 0 & 0 & 0 & 0 & 0 & 0 & 0 & 0 & 0 & 0 \\
\hline$\left\langle P_{M 1}\right|$ & $-V_{s 0 p 1} h_{p}$ & 0 & $E_{p 1}$ & 0 & 0 & 0 & 0 & 0 & 0 & 0 & 0 & 0 & 0 & 0 & 0 & 0 \\
\hline$\left\langle S_{1}\right|$ & 0 & 0 & 0 & $E_{S 1}$ & 0 & $-V_{s 1 p 0} h_{x}$ & 0 & 0 & 0 & 0 & 0 & 0 & 0 & 0 & 0 & 0 \\
\hline$\left\langle S_{2}\right|$ & 0 & 0 & 0 & 0 & $E_{S 1}$ & 0 & $-V_{s 1 p 0} h_{y}$ & 0 & 0 & 0 & 0 & 0 & 0 & 0 & 0 & 0 \\
\hline$\left\langle X_{0}\right|$ & 0 & 0 & 0 & $V_{s 1 p 0} h_{x}$ & 0 & $E_{p 0}$ & 0 & $V_{p p \pi} g_{z}$ & 0 & 0 & 0 & 0 & 0 & 0 & 0 & 0 \\
\hline$\left\langle Y_{0}\right|$ & 0 & 0 & 0 & 0 & $V_{s 1 p 0} h_{y}$ & 0 & $E_{p 0}$ & 0 & $V_{p p \pi} g_{z}$ & 0 & 0 & 0 & 0 & 0 & 0 & 0 \\
\hline$\left\langle X_{3}\right|$ & 0 & 0 & 0 & 0 & 0 & $V_{p p \pi} g_{z}$ & 0 & $E_{p 1}$ & 0 & 0 & 0 & 0 & 0 & 0 & 0 & 0 \\
\hline$\left\langle Y_{3}\right|$ & 0 & 0 & 0 & 0 & 0 & 0 & $V_{p p \pi} g_{z}$ & 0 & $E_{p 1}$ & 0 & 0 & 0 & 0 & 0 & 0 & 0 \\
\hline$\left\langle Z_{3}\right|$ & 0 & 0 & 0 & 0 & 0 & 0 & 0 & 0 & 0 & $E_{p 1}$ & $V_{p p \sigma} g_{z}$ & 0 & 0 & 0 & 0 & 0 \\
\hline$\left\langle Z_{0}\right|$ & 0 & 0 & 0 & 0 & 0 & 0 & 0 & 0 & 0 & $V_{p p \sigma} g_{z}$ & $E_{p 0}$ & 0 & 0 & 0 & 0 & 0 \\
\hline$\left\langle P_{M 2}\right|$ & 0 & 0 & 0 & 0 & 0 & 0 & 0 & 0 & 0 & 0 & 0 & $E_{p 1}$ & 0 & 0 & 0 & 0 \\
\hline$\left\langle P_{M 3}\right|$ & 0 & 0 & 0 & 0 & 0 & 0 & 0 & 0 & 0 & 0 & 0 & 0 & $E_{p 1}$ & 0 & 0 & 0 \\
\hline$\left\langle P_{M 4}\right|$ & 0 & 0 & 0 & 0 & 0 & 0 & 0 & 0 & 0 & 0 & 0 & 0 & 0 & $E_{p 1}$ & 0 & 0 \\
\hline$\left\langle Y_{1}\right|$ & 0 & 0 & 0 & 0 & 0 & 0 & 0 & 0 & 0 & 0 & 0 & 0 & 0 & 0 & $E_{p 1}$ & 0 \\
\hline$\left\langle X_{2}\right|$ & 0 & 0 & 0 & 0 & 0 & 0 & 0 & 0 & 0 & 0 & 0 & 0 & 0 & 0 & 0 & $E_{p 1}$ \\
\hline
\end{tabular}


Supporting information 2: Alternative values for the TB parameters (eV) and computed Absorption

\begin{tabular}{ccccccccccc}
\hline \hline$V_{S S}$ & $V_{s 0 p 1}$ & $V_{p 0 s 1}$ & $V_{p p \sigma}$ & $V_{p p \pi}$ & $E_{s 0}$ & $E_{s 1}$ & $E_{p 0}$ & $E_{p 1}$ & $\Delta_{s o 0}$ & $\Delta_{s o 1}$ \\
\hline-1.10 & 1.25 & 0.60 & -3.50 & 0.80 & -9.21 & -13.21 & 2.39 & -2.16 & 1.30 & 1.20
\end{tabular}

Table S1. Tight binding parameters for $\mathrm{MAPbI}_{3}$ band diagram with SOC, $m_{h}^{*}=0.235 m_{0}$ for the valence band and $m_{e}^{*}=0.229 m_{0}$ for the conduction band and $E_{G}=1.620 \mathrm{eV}$.
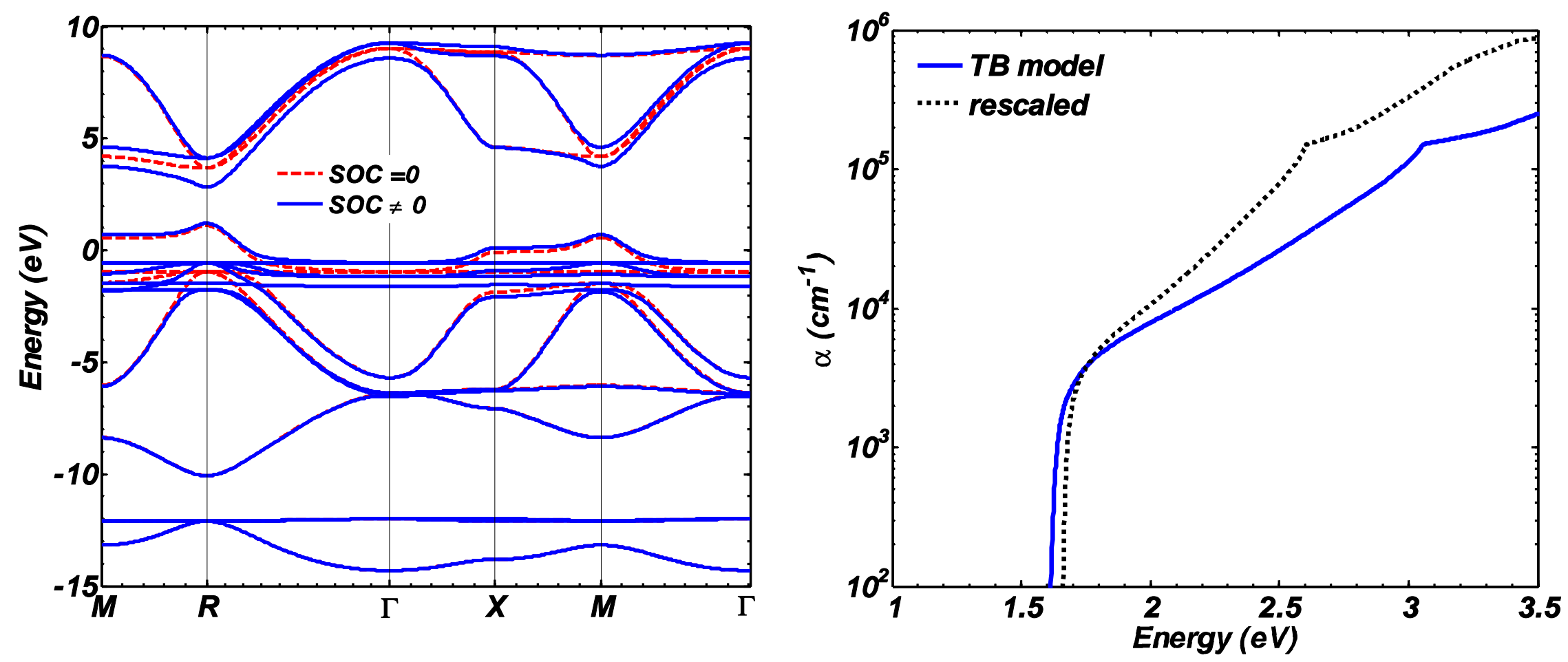

Figure S1. Computed properties of $\mathrm{MAPbI}_{3}$ with the TB parameters of Table S1: (left panel) band diagram including SOC (blue line) or not (red dotted line) (right panel) absorption spectra (including SOC) computed within the TB model (blue line) and after rescaling the energy axis (dotted line) according to the scaling procedure: $\mathrm{h} v \rightarrow 0.653 \mathrm{~h} v+0.608$ proposed in Ref. 52 . 


\section{Supporting information 3: Effect of $p(X)$ spin-orbit coupling}

Figure S2 presents the effect of $\Delta_{\mathrm{so} 1}$ on the band diagram.
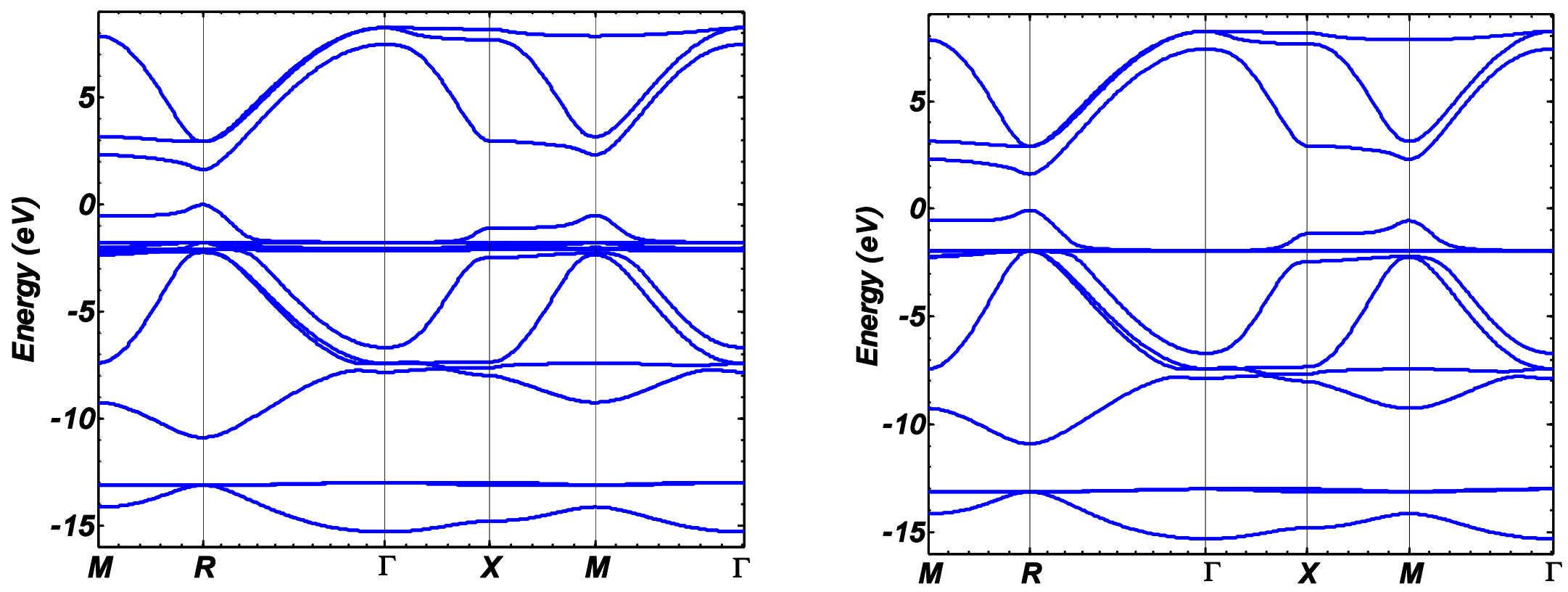

Figure S2. $\mathrm{MAPbI}_{3}$ band diagrams obtained with Table 1 parameters except $\Delta_{s o 1}$. Left side : $\Delta_{s o 1}=0.45 \mathrm{eV}$. Right side $: \Delta_{s o 1}=0 \mathrm{eV}$

A smaller value of $\Delta_{s o 1}$ reduces the dispersion of $p(\mathrm{X})$ bands. 10 of them are totally flat with $\Delta_{s o 1}=0$. It also increases slightly the bandgap and the reduced effective mass. Indeed, $E_{G}=1.65 \mathrm{eV}$ and $\mu=0.111 \mathrm{~m}_{0}$ with $\Delta_{s o 1}=0.45 \mathrm{eV}$, and $E_{G}=1.68 \mathrm{eV}$ and $\mu=0.112 \mathrm{~m}_{0}$ for $\Delta_{s o 1}=0 \mathrm{eV}$. 